\title{
INDICADORES
}

\section{RANKING UNIR, UN PROYECTO DE CLASIFICACIÓN DE LA MEJOR PUBLICIDAD IBEROAMERICANA}

\section{UNIR: a project of classification of the best Latin American advertising}

\section{Rafael Repiso e Inmaculada Berlanga}

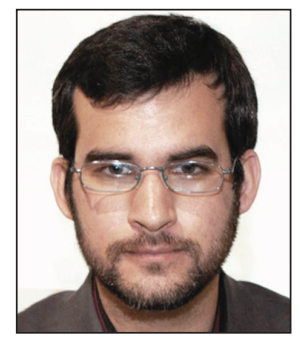

Rafael Repiso es diplomado y licenciado en biblioteconomía y documentación, master en evaluación científica y doctor en documentación por la Universidad de Granada. Profesor de la Universidad Internacional de la Rioja (UNIR) desde 2013, es miembro de los grupos de investigación EC3 (Universidad de Granada) y Procomm (UNIR). Socio fundador de la spin off EC3metrics. Su línea de investigación gira en torno al uso de métodos cuantitativos en documentación y comunicación. http://orcid.org/0000-0002-2803-7505

Universidad Internacional de la Rioja Gran Vía Rey Juan Carlos I, 41. 26002 Logroño, España rafael.repiso@gmail.com

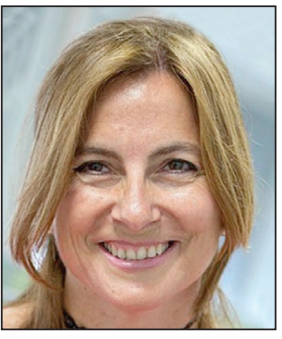

Inmaculada Berlanga es licenciada y doctora en filología, y licenciada y doctora en comunicación audiovisual por la Universidad de Granada. Es profesora agregada en la Universidad Internacional de la Rioja (UNIR). Visiting professor en la Università Cattolica del Sacro Cuore (Milano). Es directora de Procomm (Prospectivas en Comunicación Multimedia), de UNIR Research. http://orcid.org/0000-0002-0135-624X

Universidad Internacional de la Rioja Gran Vía Rey Juan Carlos I, 41. 26002 Logroño, España inmaculada.berlanga@unir.net

\section{Resumen}

El Ranking UNIR es un meta-producto que evalúa la calidad de los anuncios y agencias de Iberoamérica a través de los premios que reciben los anuncios en festivales de publicidad nacionales e internacionales.El proyecto aparece como novedad debido al vacío de rankings publicitarios en el contexto iberoamericano. Cuenta con tres espacios fundamentales: el Ranking Iberoamericano de Publicidad y los sub-rankings nacionales; un repositorio de los mejores anuncios de la publicidad iberoamericana, y un directorio de agencias de publicidad. Estas tres dimensiones lo convierten en un producto académico idóneo para la investigación de la publicidad en Iberoamérica y para la docencia universitaria.

\section{Palabras clave}

Publicidad; Iberoamérica; Rankings; Ranking de publicidad; Agencias de publicidad; Anuncios publicitarios; Festivales de publicidad; Premios de publicidad.

\section{Abstract}

The UNIR Ranking is a meta-product that evaluates the quality of ads and advertising agencies in Latin America through the awards made in national and international advertising festivals. The project is a novelty due to the vacuum of advertising rankings in the Latin American context. It has three main activity areas: the Iberoamerican advertising ranking and subnational rankings, a repository of the best adverts of the Latin American geographical area, and a directory of Latin American publicity agencies. These three dimensions make the UNIR Ranking an ideal product for academic research on Latin American advertising activities as well as for university teaching.

\section{Keywords}

Advertising; Latin America; Rankings; Advertising rankings; Advertising agencies; Advertising festivals; Advertising awards. 
Repiso, Rafael; Berlanga, Inmaculada (2015). "Ranking UNIR, un proyecto de clasificación de la mejor publicidad iberoamericana". El profesional de la información, v. 24, n. 4, pp. 479-484.

http://dx.doi.org/10.3145/epi.2015.jul.15

\section{Ranking UNIR, un producto renovado}

El Ranking UNIR de publicidad es un producto académico que forma un catálogo ordenado de anuncios y agencias de publicidad para Iberoamérica de acuerdo a criterios previamente establecidos. En él se ponderan los premios recibidos en medio centenar de festivales de publicidad, internacionales e iberoamericanos. Tiene su origen en el producto limitado a España Ranking ESCO de agencias de publicidad (Repiso; Llorente-Barroso; García-García, 2013), que absorbe e incorpora. Aunque este último parte de la misma idea del anterior de evaluar las agencias y los anuncios a través de los premios que han recibido en festivales, se ha incrementado considerablemente el nivel de agregación (tabla 1). El Ranking UNIR pretende suplir la carencia de rankings de publicidad en los principales países iberoamericanos, en conjunto y por sectores nacionales. Existe otro producto similar en Iberoamérica, el Ranking Crema de Adlatina, pero limitado en cuanto a número de elementos (Wentz, 2009), puesto que sólo muestra el top ten de cada país.

Además de ranking, es un repositorio de anuncios y un listado de las principales agencias de publicidad iberoamericanas, de las que también se pueden consultar datos identificativos, como los anuncios premiados y los premios que han recibido. La actualización de datos se lleva a cabo una vez al año, empieza en enero y termina en mayo.

Los rankings de empresas (agencias) y anuncios son muy comunes en el sector publicitario por la propia dinámica competitiva del sector. Nacieron en Estados Unidos en los años sesenta (Kübler; Proppe, 2012) y se desarrollaron principalmente en el mundo anglosajón. Los festivales publicitarios son, además de un espacio de promoción para las agencias publicitarias, un producto con elementos comunes a los rankings, ya que establecen clasificaciones de anuncios y agencias de acuerdo a parámetros previamente concretados (Helgesen, 1994). Los rankings suelen premiar la creatividad o la eficiencia publicitaria (Montano, 2004), aunque según Helgesen, es la creatividad el principal elemento de evaluación (1994).

Desde el punto de vista metodológico esta opción está bien fundada porque:

Tabla 1. Diferencias cuantitativas entre el Ranking ESCO (2012) y el Ranking UNIR (2014)

\begin{tabular}{|l|c|c|}
\hline & Ranking ESCO & Ranking UNIR \\
\hline Premios & 909 & 10.062 \\
\hline Anuncios & 611 & 5.826 \\
\hline Agencias & 197 & 1.045 \\
\hline Festivales & 13 & 49 \\
\hline Países & 1 & 22 \\
\hline Rankings nacionales & 1 & 8 \\
\hline
\end{tabular}

- trabaja sobre valoraciones ajenas a los que confeccionan el listado; en el peor de los casos sería un procedimiento para objetivar lo subjetivo;

- incluye criterios que posibilitan la atención de muchos más aspectos de los que una valoración académica podría atender;

- se trabaja sobre valoraciones de expertos (los jueces de festivales y premios) sobre criterios previa y públicamente conocidos;

- los jueces proceden normalmente del ámbito profesional de la publicidad y eso constituye un elemento de relación con lo académico que enriquece su inclusión y el resultado final.

Este procedimiento permite el enriquecimiento progresivo del ranking con la incorporación de nuevos festivales con su adecuada ponderación. Por otra parte, la utilización de los festivales de publicidad como fuente de información para elaborar un ranking es práctica común en el mundo anglosajón. Desde 2003, los publicistas Dorte Collister y Patrick Collister realizan un meta-ranking enfocado al mundo anglosajón titulado The Directory big won rankings, publicado por la revista Directory:

https://www.directnewideas.com/bigwon

Además de ranking, UNIR es un repositorio de anuncios y un listado de las principales agencias de publicidad iberoamericanas

Si tuviésemos que comparar ambos productos destacaríamos que Big won rankings es internacional, analizando datos a partir de los premios en 32 festivales internacionales, con un fuerte sesgo anglosajón. Por el contrario el Ranking UNIR se centra exclusivamente en Iberoamérica, analizando 49 festivales internacionales e iberoamericanos. Ambos utilizan los premios como fuentes, pero el Ranking UNIR además pondera individualmente cada premio según unos criterios propios.

Hasta la aparición del Ranking UNIR no existían rankings Iberoamericanos de publicidad, aunque sí existe una comunidad publicitaria iberoamericana y se encuentra cohesionada. Lo prueban los intercambios profesionales, la existencia de productos comunes o los numerosos festivales de publicidad iberoamericanos, como el Festival El Sol, que comenzó limitado a España y evolucionó a Iberoamérica (Farrán-Teixidó; López-Lita, 2010). En cambio, cuando se analiza la publicidad iberoamericana lo habitual es que se haga desde enfoques nacionales o que directamente se salte al nivel internacional, y se pierda así la oportunidad de analizarla en su contexto regional y cultural más inmediato. 


\section{Plataforma de gestión de} premios, anuncios y agencias del Ranking UNIR

El nuevo proyecto ofrece un gestor de contenidos propio, que en conexión con una base de datos permite realizar todo el proceso de carga directamente, en una sola etapa. Anteriormente, en el Ranking ESCO los cáculos se hacían por separado. Ahora se realiza el cálculo de los indicadores automáticamente cada vez que se incorporan o modifican (correcciones) los premios de publicidad y se aprovechan así las posibilidades de una base de datos dinámica, estructurada en torno a los premios (figura 1).

La creación de una plataforma de gestión de datos ofrece muchas posibilidades al sistema: se aumenta el control, la transparencia y además permite que el producto siga creciendo e incorpore nuevos festivales y rankings. La interacción que el gestor de contenidos (tabla 2) realiza sobre la base de datos (figura 1), permitirá en un futuro crear rankings históricos o nacionales en nuevos países.

La publicidad de estos países se examina desde enfoques nacionales o internacional, con lo que se pierde el análisis de la publicidad en su contexto regional y cultural más inmediato

Por tanto presenta tres aportaciones fundamentales:

- Ranking iberoamericano de publicidad y sub-rankings nacionales;

- repositorio de los mejores anuncios de la publicidad iberoamericana;

- directorio de agencias de publicidad iberoamericana.

Todo ello le habilita para facilitar el estudio de la publicidad en Iberoamérica, especialmente en aspectos creativos y de calidad de producción de anuncios así como de visibilidad y volumen relativo de las agencias implicadas (esto último mediante indicadores indirectos).

\section{Ranking iberoamericano y sub-rankings nacionales}

El Ranking UNIR utiliza como fuente de información los premios que las agencias iberoamericanas reciben en 49 festivales nacionales, regionales (europeos e iberoamericanos) e internacionales. De esto se deriva una característica fundamental en la composición de los actores del ranking; sólo aparecen anuncios premiados en algunos de estos festivales y sólo las agencias cuyo/s anuncio/s ha/n sido premiado/s. El ranking "se alimenta" de premios.

La ponderación de los premios mantiene los criterios del Ranking ESCO. Para calcular el valor de una agencia se tienen en cuenta los premios recibidos. Cada premio tiene asignado un valor en función de la importancia y relevancia del festival en el que obtiene el premio (nacional, regional e internacional). Este valor se matiza con la posición que implica el premio (oro, plata, etc.). Para calcular el valor de un año determinado se tienen en cuenta los premios recibidos en los dos años anteriores, como hace el impact factor (Garfield, 1999). El valor de una agencia para 2014 depende de los premios recibidos en 2012 y 2013 (ecuación 1).

Tabla 2. Unidades de trabajo del sistema de gestión del Ranking UNIR de publicidad

\begin{tabular}{|l|l|}
\hline \multicolumn{1}{|c|}{ Unidades } & \\
\hline Zonas de ranking & $\begin{array}{l}\text { Permite crear nuevos rankings y definir las áreas de las que se va alimentar cada ranking/sub-ranking, y la } \\
\text { nacionalidad de las agencias que van a participar. Por ejemplo el ranking de Argentina se calcula para toda } \\
\text { agencia ganadora de premios de publicidad en festivales argentinos o bien para toda agencia argentina que } \\
\text { haya ganado premios en festivales regionales o internacionales. }\end{array}$ \\
\hline Festivales & $\begin{array}{l}\text { Permite incorporar o borrar festivales, así como asignarles una zona geográfica determinada (nacional, regio- } \\
\text { nal e internacional) y el valor correspondiente a cada festival }(1 ; 1,5 ; 2 ; \text { o } 2,5) .\end{array}$ \\
\hline Agencias de publicidad & Permite añadir, eliminar, modificar o combinar agencias duplicadas. \\
\hline Anuncios & Permite añadir, eliminar, modificar o combinar anuncios duplicados. \\
\hline Premios & Permite añadir, eliminar o modificar premios de publicidad. \\
\hline Usuarios identificados & $\begin{array}{l}\text { Permite la gestión de usuarios identificados con diferentes permisos para la intranet del producto: ver, editar, } \\
\text { añadir, eliminar datos o buscar dentro de la base de datos. }\end{array}$ \\
\hline
\end{tabular}




$$
\text { Agencia }_{z}=\frac{\sum_{i=1}^{m} \sum_{j=1}^{n} \frac{F_{i}}{A n_{j}}+\sum_{x=1}^{p} \sum_{y=1}^{q} \frac{F_{x}}{A n_{y}}{ }^{1 / 2}}{\left(\text { Agencia }_{\alpha}\right)^{1 / 2}}
$$

\author{
Valor normalizado de la agencia " $z$ " para 2014: \\ z: agencia evaluada \\ $\mathrm{m}$ : festival \\ n: posición del anuncio dentro del festival \\ Fi: valor del festival en 2012 \\ Anj: valoración del premio recibido en el festival en 2012 \\ $p$ : festival \\ q: posición del anuncio dentro del festival \\ Fx: valor del festival en 2013 \\ Any: valoración del premio recibido en el festival en 2013
}

Ecuación 1. Fórmula del ranking de agencias del Ranking UNIR

Con los anuncios se utiliza una fórmula casi idéntica con una modificación: en el ranking de anuncios de un año, por ejemplo de 2013, sólo se tienen en cuenta los premios recibidos en 2013, por tanto se tiene en cuenta un período de un año y se calcula sobre ese mismo año, de forma similar al immediacy index de las revistas (Tomer, 1986). Esto da como resultado que muchos anuncios aparezcan en los rankings de anuncios de varios años. Pensemos que un anuncio que se crea en octubre de 2012 puede ser premiado en festivales de 2012, en festivales de 2013 y quizás en alguno de 2014, por lo que ese anuncio estaría presente en los rankings de anuncios de 3 años.

Para cada año hay dos listados: un ranking de agencias y otro de anuncios. Ambos tienen como fuente los premios en festivales de publicidad. Por tanto el Ranking clasifica tanto agencias como anuncios.

\title{
Rankings nacionales (sub-rankings)
}

Se calculan en aquellos países con una masa crítica de premios suficientes para dar consistencia a cada clasificación, al menos para las 20 primeras posiciones. Actualmente sólo se calculan para Argentina, Brasil, Chile, Colombia, Ecuador México, Perú y España, donde se incorporan los de 2012 y 2013 del Ranking ESCO. Para ello se utilizan los premios recibidos en los festivales del país concreto, así como los recibidos por las agencias de ese país en festivales iberoamericanos e internacionales. En el caso de España también se tienen en cuenta festivales europeos.

\section{Ranking iberoamericano}

Se tienen en cuenta sólo los premios recibidos en festivales ibe- roamericanos e internacionales, obviando los premios en festivales nacionales, con el objetivo de medir un escenario en el que todos los países estén en igualdad de condiciones.

\section{Repositorio de los mejores anuncios de publicidad iberoamericana}

La fuente de datos son los festivales cuya primera misión, antes que la ponderativa, es la de difundir los mejores trabajos del sector publicitario, con especial hincapié en los aspectos creativos (Kübler; Proppe, 2012).

El ranking hereda también esta dimensión de los festivales y se convierte en un repositorio de facto cuando, a través de los rankings o las agencias podemos ver la colección de los mejores anuncios de una agencia, un país o Iberoamérica en el período de cobertura del producto, visualizando las piezas de la mayoría de ellos gracias a vídeos incrustados.

\section{Para calcular el valor de una agencia en un año determinado se toman en cuen- ta los premios recibidos en los dos años anteriores, como hace el impact factor}

Cuando se selecciona un anuncio, la plataforma conduce a un apartado donde se aportan sus principales datos identificativos (nombre, agencia productora, país). Además, el sistema muestra la estadística de la posición contextualizada de los anuncios en los rankings en los que participa y en los años en los que ha sido premiado. Desde este apartado se pueden localizar los premios que ha recibido cada anuncio.

\section{Directorio de agencias de publicidad iberoamericana}

Es también un buen directorio de las agencias iberoamericanas, limitado a aquellas que han sido premiadas en los

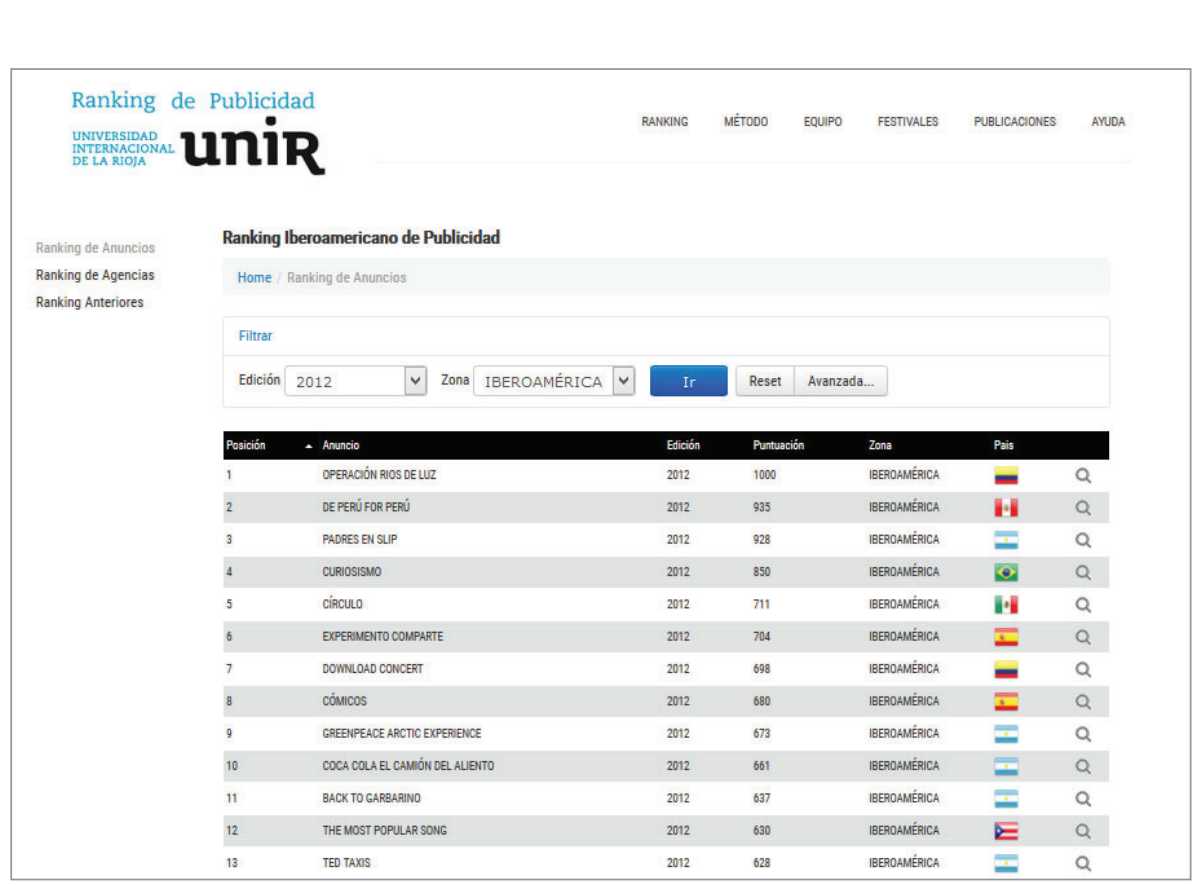

Imagen 1. Ranking de anuncios iberoamericano para 2012 
festivales fuente. Además de incluir los principales datos identificativos de las agencias (dirección, web, responsable, contacto, etc.), permite ver, como si fuese una sala de trofeos, los anuncios premiados de cada agencia, así como los premios. Este hecho incorpora una información muy útil sobre las agencias, las temáticas en las que trabajan y las formas que utilizan para desarrollar sus campañas publicitarias.

Para el ranking de anuncios se tiene en cuenta un período de un año y se calcula sobre ese mismo año, de forma similar al immediacy index de las revistas

\section{Herramienta de estudio de la publicidad iberoamericana}

El Ranking UNIR se planteó desde su comienzo como una herramienta científica que permitiese identificar los mejores anuncios y las mejores agencias de publicidad de toda Iberoamérica. El objetivo -mediante la identificación, ordenación, clasificación y ponderación de los anuncios y agencias- es aportar luz sobre aspectos que permitan estudiar la publicidad del ámbito y de cada uno de los países iberoamericanos, así como describir y estudiar las relaciones. Como ejemplo, el Ranking permite identificar los países con más peso en la publicidad iberoamericana (figura 2). Muestra asimismo cómo son las agencias de publicidad multinacionales las que acaparan las primeras posiciones de todos los rankings, lo que Fejes (1980), analizando la publicidad en Latinoamérica a principios de los ochenta, llamó "dominio mundial norteamericano de los medios de comunicación".

Posibilita también localizar y analizar temas, justificar muestras de estudio, realizar análisis comparativos en la región, etc. Además, puede emplearse como recurso didáctico para encontrar fácilmente piezas que ilustren las clases de los profesores universitarios.
Como objetivos inmediatos se pretende:

- mejora continua de la plataforma;

- implementación de los buscadores internos;

- enriquecimiento de la información que se aporta sobre anuncios y agencias.

Igualmente se aumentará el número de festivales (la próxima edición contará con 52 festivales publicitarios), para poder construir rankings nacionales fiables en aquellos países de Iberoamérica de los que aún no existe información suficiente.

El propósito del proyecto actual es la mejora continua de la plataforma implementando los buscadores internos y enriqueciendo la información que se aporta sobre anuncios y agencias

\section{Agradecimientos}

Este proyecto es deudor de muchos investigadores que lo han mejorado con sus aportaciones. Destacamos a Francisco García y Carmen Llorente quienes desde un principio han estado involucrados en el mismo, así como al equipo técnico y a los miembros del comité asesor.

Nuestro agradecimiento a Julio Montero por su colaboración con el proyecto buscando los recursos necesarios para su creación y actualización, y por su colaboración en la redacción y revisión de estilo de este trabajo.

Investigación financiada por el plan propio de investigación de la Universidad Internacional de la Rioja (UNIR), bienio 2014-2015. Gdl-13. Procomm.

\section{Bibliografía}

Farrán-Teixidó, Eduard; López-Lita, Rafael (2010). “La evolución del discurso racional al discurso emocional en El Sol. El festival iberoamericano de la comunicación publicitaria

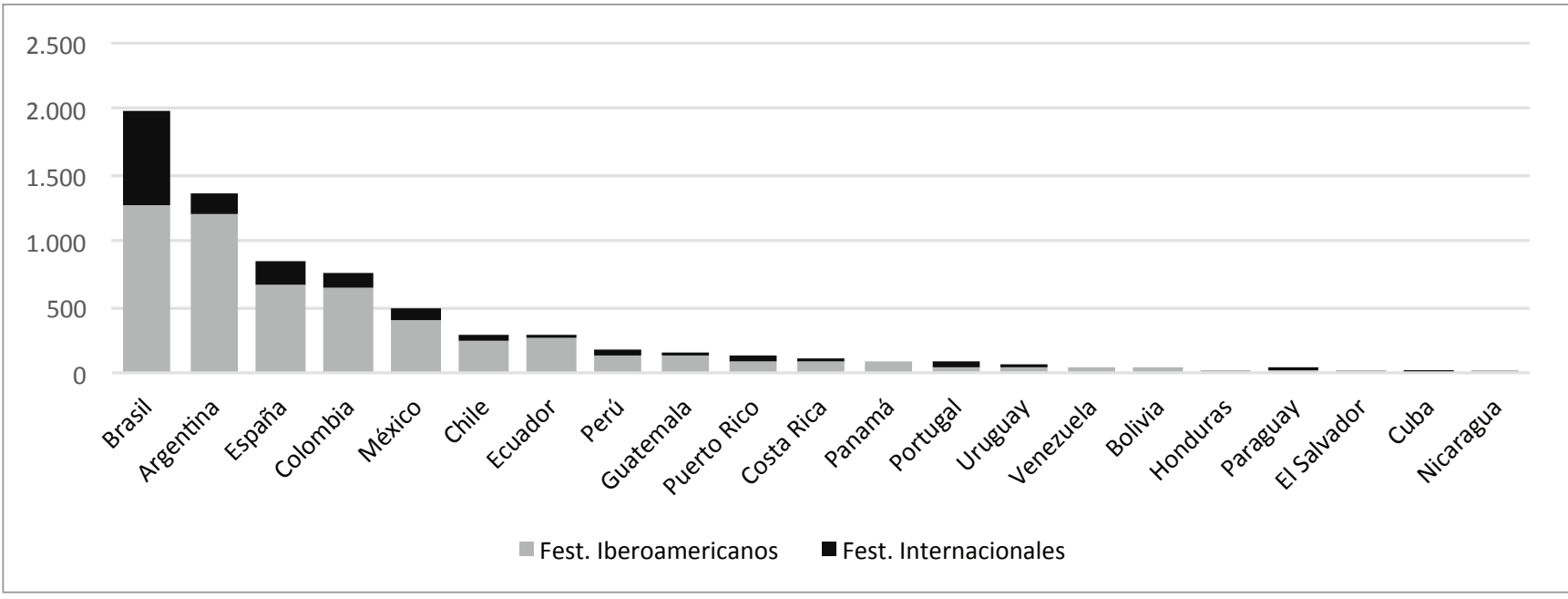

Figura 2. Distribución de premios de publicidad en festivales por países según el Ranking UNIR 2012-2013 
(1988-1998-2008)". Comunicación y hombre, n. 6, pp. 43-63. http://www.comunicacionyhombre.com/articulo. php?articulo $=22$

Fejes, Fred (1980). "The growth of multinational advertising agencies in Latin America". Journal of communication, v. 30, n. 4, pp. 36-49.

http://dx.doi.org/10.1111/j.1460-2466.1980.tb02014.x

Garfield, Eugene (1999). "Journal impact factor: a brief review". Canadian Medical Association Journal (CMAJ), v. 161, n. 8, pp. 979-980.

http://www.ncbi.nlm.nih.gov/pmc/articles/PMC1230709/ pdf/cmaj_161_8_979.pdf

Helgesen, Thorolf (1994). "Advertising awards and advertising agency performance criteria". Journal of advertising research, v. XXXIV, n. 4, July/August, pp. 43-53.

Kübler, Raoul V.; Proppe, Dennis (2012). "Faking or convincing: Why do some advertising campaigns win creativity awards?". Business research, v. 5, n. 1, pp. 60-81. http://link.springer.com/article/10.1007/BF03342732
Montano, Jesse-Raúl (2004). Characteristics of US Hispanic advertising. A comparison of award-winning and non-award winning commercials. Thesis. University of Florida.

http://etd.fcla.edu/UF/UFE0005381/montano_j.pdf

Repiso, Rafael; Llorente-Barroso, Carmen; García-García, Francisco (2013). "Ranking ESCO de agencias de publicidad: descripción y resultados". El profesional de la información, v. 22 , n. 2 , pp. 181-187.

http://dx.doi.org/10.3145/epi.2013.mar.13

Tomer, Christinger (1986). "A statistical assessment of two measures of citation: The impact factor and the immediacy index". Information processing \& management, v. 22, n. 2, pp. 251-258.

http://dx.doi.org/10.1016/0306-4573(86)90057-9

Wentz, Laurel (2009). "Almap BBDO reigns again as the most-awarded shop in Latin America". Advertising age, September 30.

http://adage.com/article/global-news/ad-show-adlatina-screma-ranks-almap-bbdo-awarded-shop/139363

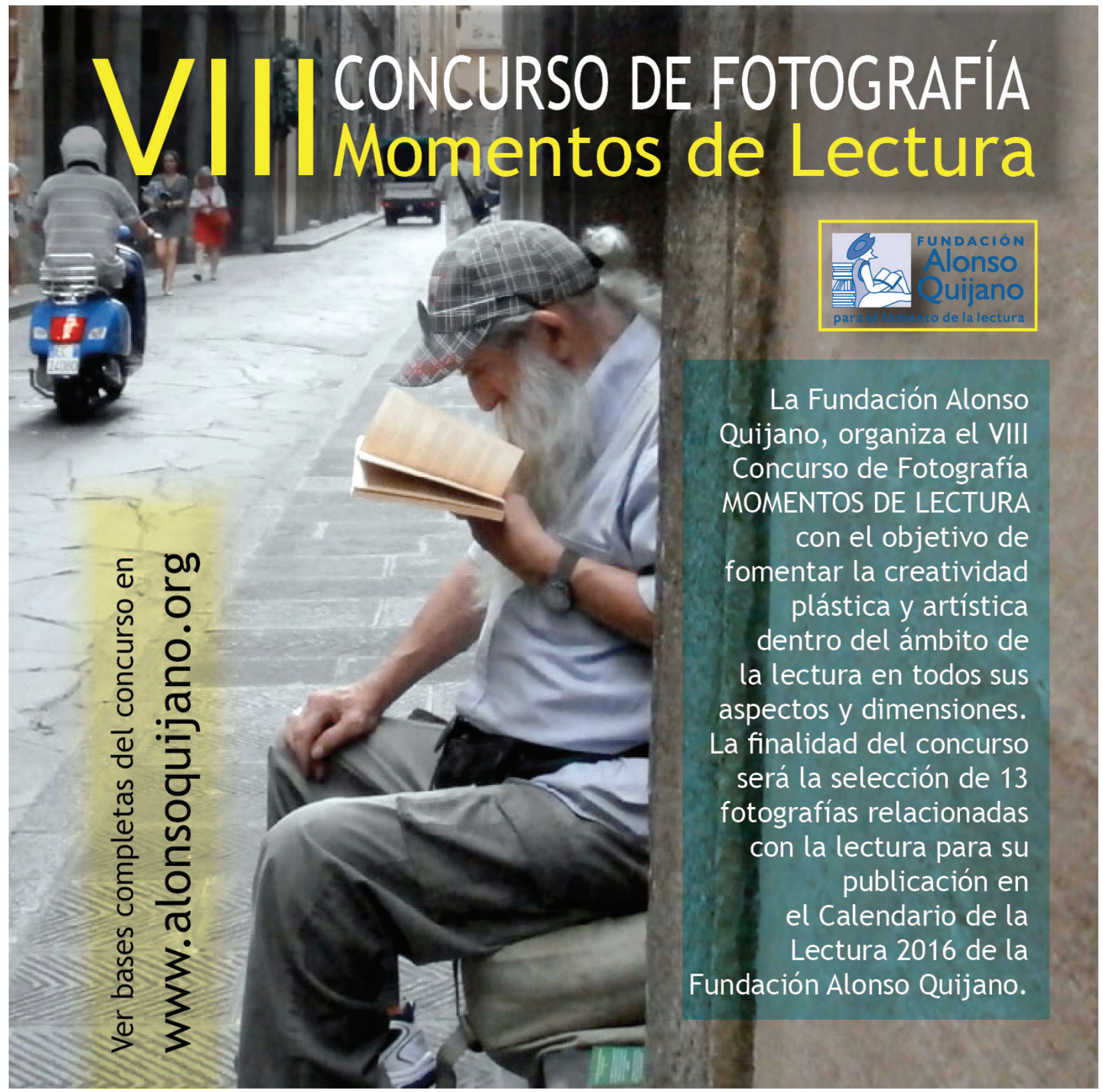

